\title{
A case of Japanese encephalitis misdiagnosed as tuberculous meningoencephalitis
}

\section{YanJun Liu ( $\sim$ liuyanjunlzu@163.com )}

Lanzhou University Second Hospital https://orcid.org/0000-0001-6075-6836

\section{MingMing Li}

Lanzhou University Second Hospital

Jie Li

Lanzhou University Second Hospital

Bin Qiang Ye

Lanzhou University Second Hospital

\section{Case report}

Keywords: Japanese encephalitis,tuberculous meningoencephalitis,central nervous system infection disease,misdiagnosis

Posted Date: March 11th, 2019

DOI: https://doi.org/10.21203/rs.2.438/v1

License: (1) (1) This work is licensed under a Creative Commons Attribution 4.0 International License. Read Full License 


\section{Abstract}

Backgroud Japanese encephalitis is a kind of central nervous system infectious disease caused by Japanese encephalitis virus transmitted through mosquito[1].Most patients with JE are acute onset and critically ill, with high fever, disturbance of consciousness, epilepsy, abnormal mental behavior, cognitive impairment, and positive meningeal irritation. Case presentation A 15-year-old boy was admitted to the hospital with acute febrile illness, headache,jet vomiting,disorder of consciousness,diagnosed as central nervous system (CNS) infection. According to the characteristics of blood and cerebrospinal fluid (CSF), the patient was initially diagnosed with meningoencephalitis and took antituberculosis treatment.However,subsequent magnetic resonance images(MRI) did not match the performance of typical tuberculous meningoencephalitis.Further laboratory testing for infections was conducted. JE was confirmed by the Disease Control Center of Lanzhou City through the detection of Japanese encephalitis $\operatorname{lgM}$ antibodies in both blood and CSF of the patients.After diagnosis of JE, the patient was provided the treatment of Human immunoglobulin and methylprednisolone therapy.After 3 weeks, the patient was transferred to the department of rehabilitation medicine to continue treatment. After 3 months and 6 months of follow-up, the patient recovered and the FIM score were 126, which were completely independent. Conclusion Given the similar clinical manifestations of Japanese encephalitis and other types of encephalitis, it is essential to enhance the awareness of Japanese encephalitis.

\section{Background}

JEV is a kind of zoonotic flavivirus that spreads between birds and pigs,mainly occurs in summer and autumn,which leads to JE in Southeast Asia.In the case of human infection with JEV,the virus rapidly causes central nervous system infectious diseases, which seriously threaten human health. The course of JE can be divided into three periods[2]:囚prodromal period:manifested as fever,headache, nausea and

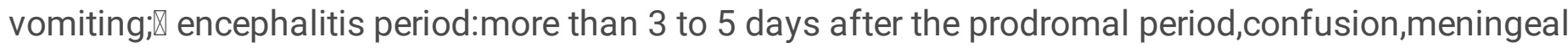
irritation,muscle rigidity,dyskinesia,etc.;》recovery period or sequelae.From the clinical manifestations,JE and other types of intracranial infections are difficult to identify,and the diagnosis of JE relies mainly on serological and pathogenic examinations.

\section{Case Presentation}

A14-years-old boy was referred to our hospital with symptoms of fever,headache for 5 days,disorder of consciousness for 1 day on August 7,2017 from Tongwei County of Gansu Province,whose diagnosis considers fever to be investigated and central nervous system infectious diseases.At that time, we considered central nervous system infectious diseases.During physical examination,the patient had fever $\left(38.8^{\circ} \mathrm{C}\right)$ and somnolence. The blood pressure,heart rate and respiratory were normal.The neck of patient was stiff,and the Kernig sign was positive.

The initial laboratory investigation at admission displayed white blood cells were7.69×109/L (normal range: $4-10 \times 109 / \mathrm{L}$ ) and neutrophil ratio wae 0.69 (normal range:0.50-0.70). Liver and kidney function 
were normal.Erythrocyte sedimentation rate was normal,but C-reactive protein was $8.71 \mathrm{mg} / \mathrm{L}(\mathrm{normal}$ range:0.00-8.20mg/L). Subsequently, a lumbar puncture was conducted and the test of CSF was performed.The CSF appeared colorless and transparent, with an initial pressure of $130 \mathrm{cmH} 20$. The white cells of CSF were $19 \times 106 / \mathrm{L}$ (normal range:0-8×106/L). Pandy test was positive.Meanwhile,the protein was $0.55 \mathrm{~g} / \mathrm{L}$ (normal Range:0.24-0.44g/L), glucose was $3.4 \mathrm{mmol} / \mathrm{L}$ (normal range:2.4-4.4mmol/L), chlorine was $112.4 \mathrm{mmol} / \mathrm{L}$ (normal range: $119-129 \mathrm{mmol} / \mathrm{L}$ ) in the CSF.The acid resistant dyeing test and ink dyeing test were negative.The detection of nine respiratory infection pathogens of blood were negative,including Legionella pneumophila,Mycoplasma pneumoniae,Chlamydia pneumoniae,Q-

Herlikectin,Adenovirus,Respiratory syncytial virus,Influenza A virus,Influenza B virus,Parainfluenza virus.Fat Darney reaction and external Fiji test were negative.ANA spectrum-ANCA-ANA-ds-DNA test were negative.Tuberculosis T cells Detection:Tuberculosis infection T cell spot detection A 6.0 SFCs510*5PBMC,tuberculosis infection T cell spot detection B 4.0 SFCs510*5PBMC.

On the 2nd day after admission,the brain MRI of patient displayed bilateral thalamus and left frontal lobe showed a low signal on T1-weighted images,a high signal on T2-weighted images,a high signal on fluidattenuated inversion recovery,a high signal on diffusion weighted imaging,and infectious diseases were considered(as shown in figure $1 \mathrm{~A}$ to $\mathrm{H}$ ). The electroencephalogram of patient showed that there were moderate abnormality with diffuse slow wave in the whole brain.Combined with above examination results,tuberculous meningoencephalitis is considered to be non-exclusive.To confirm the source of tuberculosis infection,the patient was applied further chest CT,which prompted multiple nodules in both lungs,bilateral pleural localized hypertrophy,and more consideration of tuberculosis(as shown in figure 3A).Subsequently, the patient was taken the following treatment:Anti-tuberculosis (rifampicin $0.45 \mathrm{~g}$ oral qd,isoniazid $600 \mathrm{mg}$ intravenous drip qd,ethambutol hydrochloride $0.75 \mathrm{~g}$ oral qd,pyrazinamide $1 \mathrm{~g}$ oral qd) and nutritional nerve(Cyphocholine sodium $0.75 \mathrm{~g}$ intravenous drip qd), lowering the skull pressure (mannitol $125 \mathrm{ml}$ intravenous drip q12h), sedation (olanzapine $5 \mathrm{mg}$ oral qn), anti-epilepsy (levizalacetin $0.5 \mathrm{~g}$ oral bid), liver protection (reducing glutathione $1.2 \mathrm{~g}$ intravenous drip qd), cooling and prevention treatment such as complications.

After usage of anti-tuberculosis drugs,the patient had irritability and persistent fever.Combined with the patient's medical history,the onset season and the characteristics of CSF and MRI,the second test of CSF was performed immediately. The CSF appeared colorless and transparent,with an initial pressure of $150 \mathrm{cmH} 20$. The white blood cells of CSF were $34 \times 106 / \mathrm{L}$ and mononuclear cell percentage was $96 \%$.Pandy test was positive.Meanwhile,the protein was $0.47 \mathrm{~g} / \mathrm{L}$ (normal Range:0.24-0.44g/L),glucose was $3.9 \mathrm{mmol} / \mathrm{L}$,chlorine was $113.1 \mathrm{mmol} / \mathrm{L}$ in the CSF.The acid resistant dyeing test and ink dyeing test were negative.The Samples of the patient's blood and CSF were sent to the center for disease control and prevention of Lanzhou City for further identification of CNS infection which detected specific IgM antibodies of JEV by using ELISA.After signing informed consent with patients and their families,we adjust the treatment plan for the patient according to the test results,as follows:Human immunoglobulin $0.4 \mathrm{~g} / \mathrm{kg} \cdot \mathrm{d}$ intravenous drip qd for 5 days,as well as methylprednisolone $500 \mathrm{mg}$ intravenous drip qd for 3 days,and gradually reduced to methylprednisolone until withdrawal (regular monitoring during medication, pay attention to prevent adverse reactions).After 3 weeks of treatment,the patient was 
conscious, the headache was relieved,the body temperature returned to normal,and there was no spontaneous language. The patient could answer questions with nodding or shaking,and then the patient was transferred to the rehabilitation medicine department of our hospital for further treatment.

Follow-up:after discharge,the patient continued to take anti-tuberculosis drugs and anti-epileptic drugs. Three months after discharge from the hospital,the patient review the brain CT:slightly hypodense of the basal ganglia and bilateral frontal lobes, calcification of left frontal lobe and right parietal(as shown in figure $2 A$ to $D$ ), and chest CT multiple lung nodules,considering inflammatory lesions, it is recommended to review after treatment(as shown in figure 3B).Six months after discharge,the third test of CSF was performed.The CSF appeared colorless and transparent,with an initial pressure of $140 \mathrm{cmH} 20$.The white blood cells of CSF were $3 \times 106 /$ L.Pandy test was negative.Meanwhile,the protein was $0.29 \mathrm{~g} / \mathrm{L}$,glucose was $3.8 \mathrm{mmol} / \mathrm{L}$,chlorine was $127.7 \mathrm{mmol} / \mathrm{L}$ in the CSF.The acid resistant dyeing test and ink dyeing test were negative. 3 months and 6 months after discharge,the function independent measure (FIM) of the patient were assessed as 126 points which were completely independent.

\section{Discussion}

$\mathrm{JE}$ is an acute infectious disease caused by JEV infection,mainly involving the brain parenchyma.JE is transmitted by vector mosquito bites,and the onset time is obviously seasonal. The peak period is mainly distributed from July to September. Gansu Province is located in the northwestern part of China,with traditional rural areas as the main. The epidemic situation is prevalent and the epidemic may be related to the high temperature and rainfall in Gansu Province from July to September in 2017[1].The patient lives in the countryside for a long time.The high temperature and heavy rain weather are suitable for the breeding of mosquitoes and the mosquito density is high. The above factors increases the risk of disease.In recent years, due to the widespread use of Japanese encephalitis vaccine,the incidence of Japanese encephalitis in children and adolescents has significantly reduced the encephalitis and adulthood of Japanese encephalitis[3].Patients with JE and tuberculosis co-infection, underlying disease and low immune levels are two risk factors for adult cases[4]. Making a detailed inquiry of the patient's medical history, the patient has no clear history of JE vaccination. Therefore, vaccination against JE is of great significance to prevent the spread of the disease.

The patient has acute onset,with headache, fever, and disturbance of consciousness as the main clinical manifestations.It is difficult to distinguish from other types of encephalitis and is often neglected and misdiagnosed.After admission, a central systemic infectious disease was pointed by the results of patient's CSF examination and brain MRI.Combined with the chest CT results of the patient,we once diagnosed tuberculous meningoencephalitis(TBM).We found that the characteristics of Cerebrospinal fluid are different between JE and tuberculous meningitis through reviewing the literature.Typical Cerebrospinal fluid examination results of JE include moderate increase of cell number(10-100mm3), mild increase of protein(50-200mg/dl) and normal glucose levels[5].However,the number of cerebrospinal fluid cells in TBM patients is mostly (100-1000)×106/L,mainly lymphocytes[6].Typically,the CSF shows a 
high CSF white cell count,which is predominantly lymphocytic,with a high protein and low CSF to blood glucose ratio[7].

In addition,with the wide application of MRI,the MRI examination of head can clearly show the lesion and pathological features, which is great significance for the auxiliary diagnosis of JE.JE is metamorphic inflammation, and the lesion is mainly located in gray matter,which is mainly affected by thalamic involvement.Pathological features of JE:in the acute phase,the pia mater and brain parenchyma are hyperemia and edema,the nerve cells are degenerated and necrotic,the Nissl body disappears, some thrombosis occurs and a small amount of bleeding occurs;in the subacute phase, nerve cells damage and diffuse glial cells hyperplasia;in the chronic phase,nerve tissue occurs focal liquefaction and form a softening lesion,part of the cerebral cortex, basal ganglia occur calcification. Therefore, the MRI of JE has some characteristic changes.A research showed that the typical severe $\mathrm{JE}$, the lesions involved mainly in the bilateral thalamus,cerebral and medial temporal lobe,and may have the involvement of temporal lobe,frontal lobe and basal ganglia[8].The lesion of JE usually shows a low signal on T1, a high signal on $\mathrm{T} 2$, and a high signal on FLAIR;after a few months of tracking,the lesion range is reduced,and T1 and T2 are low signals[5].The MRI of the patient showed characteristic thalamic lesions, providing reference information for the diagnosis of JE.

However,there is a few of discrimination between TBM and JE about etiology, pathology and imaging.TBM is a non-suppurative inflammation of the meninges caused by Mycobacterium tuberculosis.Combined with the spread of bacteria to the subarachnoid space, it can involve the pia mater,brain parenchyma,cerebrovascular and spinal cord. The development of TBM is divided into three stages:early manifestations of intermittent headache,may be accompanied by irregular hypothermia,night sweats and other symptoms of tuberculosis;in the mid-term gradually appear headache increased, severe with jet vomiting,cranial nerve disorders, the most common eye Neurological disorders;advanced: As the disease progresses,patients develop disturbance of consciousness,and some patients have varying degrees of neurological dysfunction,such as paralysis, seizures,and abnormal mental behavior.The clinical manifestations of TBM are non-specific,and their diagnosis depends on clinical manifestations,laboratory tests, and imaging studies, while,the method of diagnosis is the smear or culture of cerebrospinal fluid bacteria to find Mycobacterium tuberculosis. The neurological imaging of TBM is mainly characterized by meningeal enhancement,basal pool exudation, tuberculoma,hydrocephalus,etc., and has relative specificity[9].Intracranial tuberculosis is a chronic granuloma formed by Mycobacterium tuberculosis infecting brain parenchyma. Early tuberculoma showed equal T1 or slightly longer T1 and long T2 signals, and showed small nodular enhancement. Late tuberculoma could form a complete capsule with equal or slightly longer T1 and slightly longer T2 signals[10].

At present,JE is still based on prevention.Gamma globulin can induce inflammatory cell apoptosis;methylprednisolone inhibits inflammatory response,inhibits inflammatory factor release and synthesis, and reduces brain edema.In combination with the condition of this patient, human immunoglobulin and methylprednisolone were used for treatment,while the adverse effects of hormones were closely observed, and complications were actively prevented. 


\section{Conclusion}

The patients with JE commonly have acute encephalitis syndrome as the main clinical manifestation.For patients with lack epidemiological data and difficult clinical diagnosis, cerebrospinal fluid examination and imaging features may help JE diagnosis. However, the diagnosis of JE mainly depends on the detection of JEV IgM antibodies.In clinical work,it is necessary to strengthen the understanding of JE.At the same time, as a neurologist,it should carefully analyze the medical history,expand the diagnosis and treatment ideas, and accumulate clinical experience to prevent misdiagnosis.

\section{Declarations}

Acknowledgements

The authors are grateful to the patient for permission to publish the case report,the center for disease control and prevention of Lanzhou City for confirmation of the diagnosis.

Funding

No funding was obtained for this study

Availability of data and materials

The datasets used and analysed during the current study are available from the corresponding author on reasonable request.

Authors'contributions

LYJ was in charge of managing the data,performing the laboratory tests,writing the manuscript and reviewed the literature.LMM contributed to treated the patient and did the patient follow-up.LJ and YBQ helped to collecte the patient's clinical data.All authors read and approved the final manuscript.

Ethics approval and consent to participate

Not applicable.

Consent for publication

We obtained a confirmation statement of consent for publication from the patient's parents.

Competing interests

The authors declare that they have no competing interests.

Author details 
1 Department of Neurology, Lanzhou University Second Hospital,No.82 of Cuiyingmen, Chengguan District, Lanzhou 730030, Gansu Province, China.

2 Department of Neurology, Lanzhou University Second Hospital,No.82 of Cuiyingmen, Chengguan District, Lanzhou 730030, Gansu Province, China.

3 Department of Radiation Therapy, Lanzhou University Second Hospital,No.82 of Cuiyingmen, Chengguan District, Lanzhou 730030, Gansu Province, China.

4 Department of Radiation Therapy, Lanzhou University Second Hospital,No.82 of Cuiyingmen, Chengguan District, Lanzhou 730030, Gansu Province, China.

\section{Abbreviations}

CSF:Cerebrospinal fluid;CNS:central nervous system;JE: Japanese encephalitis; JEV: Japanese encephalitis virus;TBM:tuberculous meningoencephalitis

\section{References}

[1]TianHong Wang,YouQuan Gu,ChaoNing Zhou,et al. Clinical characteristics of 34 cases with Japanese encephalitis in adults[J]. Chin J neurol,2018,51(8):612-617.

[2]Tiroumourougane SV,Raghava P,Srinivasan S.Japanese viral encephalitis[J].Postgrad Med J,2002,78(918):205-215.

[3]FuXia Song,XuWen Sun,Bing Li,et al. Clinical characteristics of epidemic Japanese encephalitis in adults[J]. Chin J neurol,2010,43(2):118-121.

[4]Li XY,Ke BX,Chen CN,et al.First co-infection case of melioidosis and Japanese encephalitis in China[J].BMC Infect Dis,2018,18(1):452-456.

[5]Misra UK,Kalita J.Overview:Japanese encephalitis[J].Prog Neurobiol,2010,91(2):108-120.

[6]ChangTai Zhu,ZhongYi Hu. Progress in laboratory diagnosis of tuberculous meningitis[J].Chin J Antituberc,2011,33(12):822-824.

[7]Thwaites GE,Tran TH.Tuberculous meningitis:many questions,too few answers. Lancet Neurol 2005,4:160-170.

[8]Basumatary LJ,Raja D,Bhuyan D,et al.Clinical and radiological spectrum of Japanese encephalitis[J].J Neurol Sci,2013,325(1-2):15-21.

[9]Patkar D,Narang J,Yanamandala R,et al.Central Nervous System Tuberculosis: Pathophysiology and Imaging Findings[J].Neuroimaging Clinics of North America,2012,22(4):677-705. 
[10] MingMing Zheng,Hui Bu,YueLi Zou ,et al. Modified acid fast stain confirmed 125 cases of tuberculous meningitis clinical staging and head imaging analysis[J].J Apoplexy and Nervous Diseases,2017,34(2):153-156.

\section{Figures}
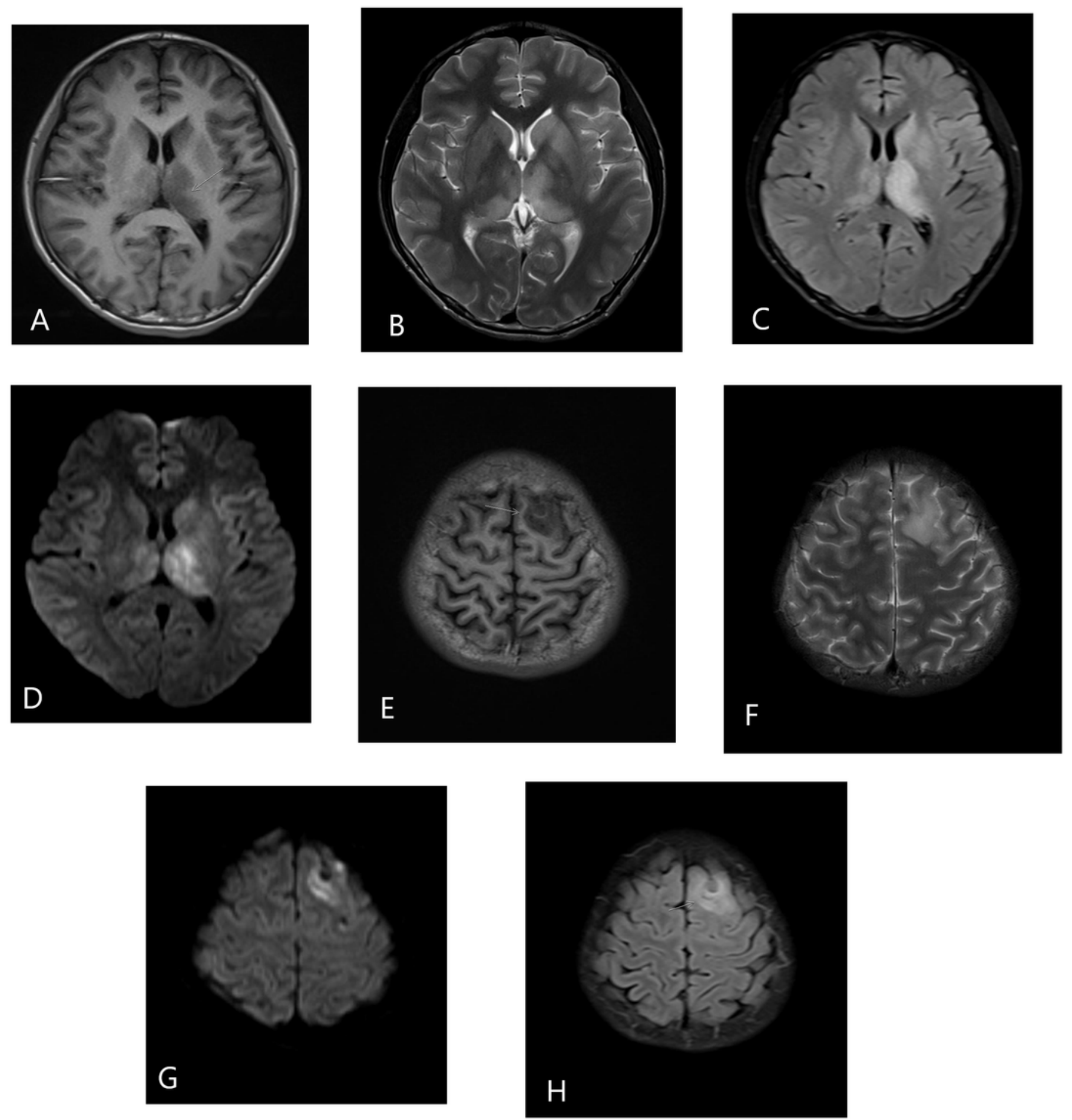

Figure 1 
Figure $1 \mathrm{~A}-\mathrm{H} \otimes \mathrm{Bilateral}$ thalamus and left frontal lobe showed a low signal on T1-weighted images,a high signal on T2-weighted images,a high signal on fluid-attenuated inversion recovery,a high signal on diffusion weighted imaging.
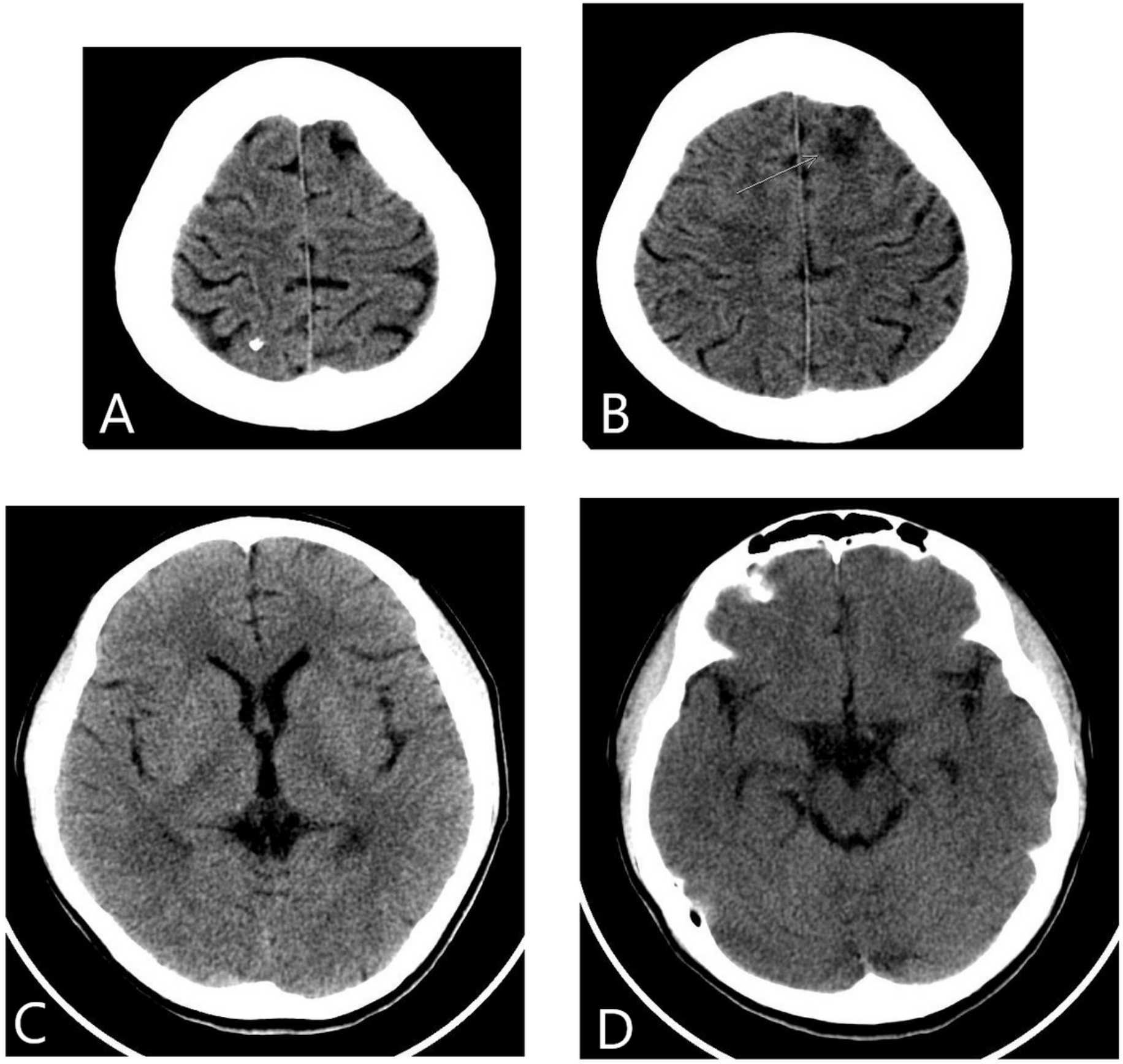

Figure 2

Figure 2A-D $₫$ Slightly hypodense of the basal ganglia and bilateral frontal lobes, calcification of left frontal lobe and right parietal. 

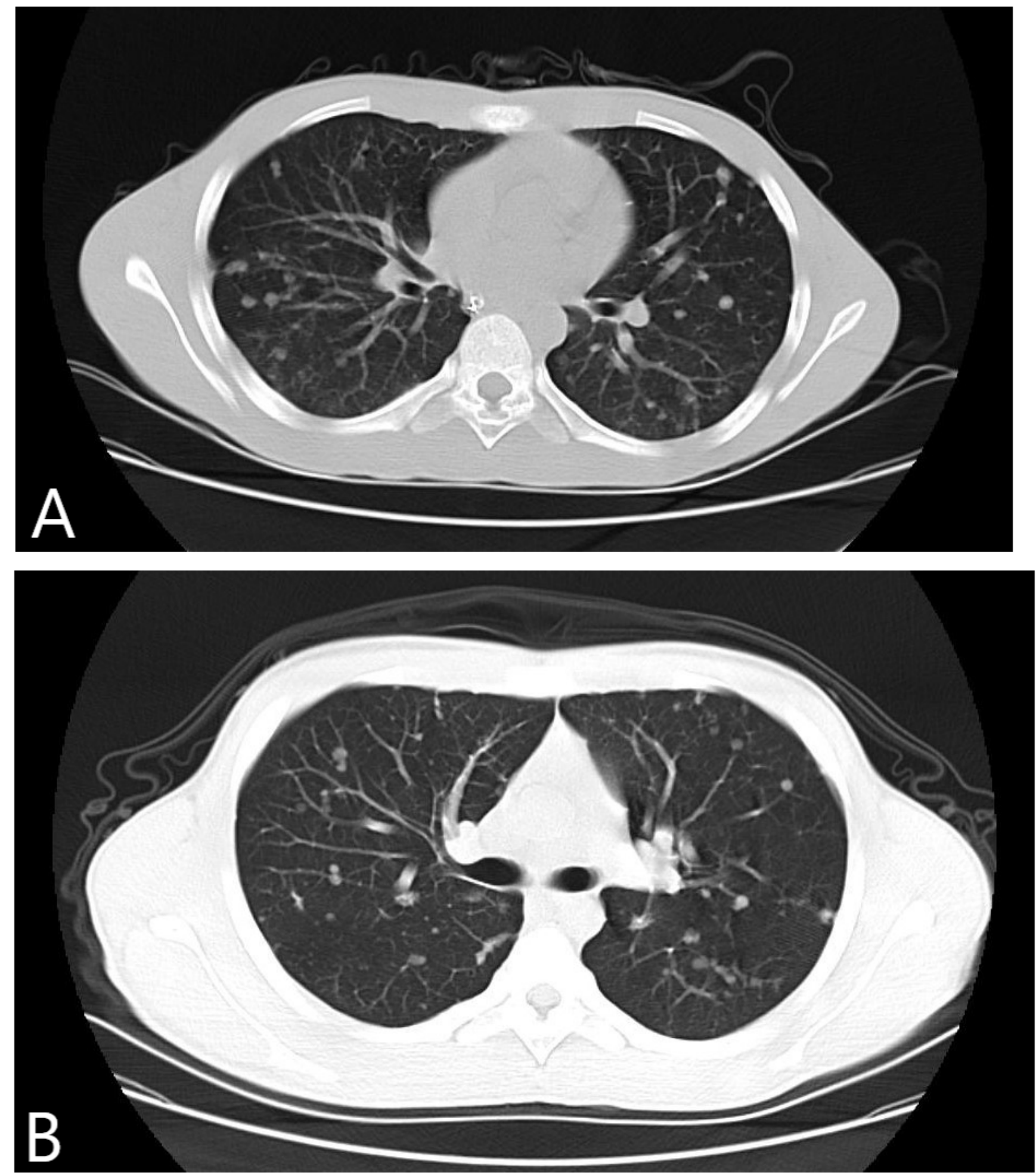

Figure 3

Figure 3A囚Multiple nodules in both lungs, bilateral pleural localized hypertrophy. Figure 3B囚Multiple nodules of in both lung. 